\section{Importance of International Exchange Programme in Postgraduate Training}

Sir,

We wish to share our views and experiences of international academic networking. An exchange programme is being established between postgraduate residency programmes of Radiation Oncology units of Pakistani and Italian universities. We wish to enhance our professional training via mutual learning in a conducive mentoring environment.

Arrangement of international collaboration during postgraduate training period is an important endeavor in order to enhance the overall strategic visioning of a training programme. Our Radiation Oncology postgraduate residency programme is a learning objectivesbased structured learning process which emphasizes on international collaborations and educational networking. We are trying to implement educational methodology, which is based on theme of 'learning via reflection'.

We wish to share our views regarding the initiation of a postgraduate educational exchange programme, which is being established between two departments of universities in Rome, Italy and Karachi, Pakistan. Modern day oncology practices are based on multidisciplinary recommendations made by members of sitespecific tumor boards. Our discipline of radiation therapy is constantly evolving with time, showing its dynamic nature. Technology plays an important role in its advancement, revolutionising it from conventional twodimensional (2D) based planning to three-dimensional (3D) CT guided planning; to introduction of beam modulation and introduction of beamlets through inverse planning calling intensity modulated radiation therapy (IMRT) to arc therapy and introduction of ion therapy. ${ }^{1}$ These advancements have allowed improvement of tumor control and decrease in side effects and toxicities due to better conformity of radiation plans allowing dose escalation to tumors and sparing organs at risk.

Newer concepts of radiomics and targeted radionuclide therapy are now new arenas to explore for response assessment, heterogeneity of tumor, altering volumes and plans for dose escalation or deescalation to stratify tumors as low and high risk of recurrence to avoid unnecessary toxicities and better response of treatment. But its validation will be justified with time.2,3 In order to keep pace with the innovations and evolution of techniques for radiation therapy, "a lifelong learner concept" is essential to identify and implement advancements in teaching and learning methodology in the field of radiotherapy for professional development. 4

In order to develop tools that allow treatment standardisation, share clinical management decisions and professional development, international linkages are required to identify the weak links and to learn from structured well-developed programmes so that bilateral mutual progress can be achieved. Furthermore, it will encourage multidisciplinary culture which is the core concept of cancer management, by involving highly specialised professionals from different centres and countries, in the form of establishing international tumor boards.

Considering these facts, a new exchange programme between Aga Khan University and Gemelli Advanced Radiation Therapy, University of Cattolica, will provide opportunities for medical education, training, research and outreach between Pakistan and Italy. The programme has been developed to create mutually beneficial collaborations between the two premier academic medical centres through bilateral exchange programmes ultimately enhancing research programmes, clinical training experiences and local healthcare.

This programme is based on learning objectives that will be fulfilled during the rotation period. The specific objectives for residents are to refine skills in clinical decision-making, to increase cross-culture competence, to understand healthcare delivery in a developing country under resource-limited settings, and appreciate unique challenges posed by this system, to improve team building and leadership skills and refine teaching techniques, gain proficiency in evidence-based medicine, improve critical appraisal skills, to acquire proficiency in basic global health issues and to learn the basic crafts of radiation therapy in the era of rapidly changing trends.

The ultimate goal of developing this exchange programme is to provide the evidence-based treatment to our patients without compromising quality and to learn facing challenges, providing it in our resource-limited settings. All these objectives can only be achieved by developing skills, doing research and developing academic collaborations with high quality international centres.

The future of cancer care lies in multidisciplinary approach; and present day postgraduate trainees will be the lead persons of site-specific multidisciplinary tumor boards of tomorrow. ${ }^{5}$ International collaborations will definitely increase our professional and academic skills. At the end of the day, it will lead to quality improvement in overall healthcare of cancer patients.

\section{REFERENCES}

1. Bucci MK, Bevan A, Roach M. Advances in radiation therapy: conventional to $3 \mathrm{D}$, to IMRT, to $4 \mathrm{D}$, and beyond. CA Cancer $J$ Clin 2005; 55:117-34. 
2. Reuzé S, Schernberg A, Orlhac F, Sun R, Chargari C, Dercle L, et al. Radiomics in nuclear medicine applied to radiation therapy: Methods, pitfalls and challenges. Int $\mathrm{J}$ Radiat Oncol Biol Phys 2018; pii:50360-3016(18)30815-0.

3. Li T, Ao EC, Lambert B, Brans B, Vandenberghe S, Mok GS. Quantitative imaging for targeted radionuclide therapy dosimetry-technical review. Theranostics 2017; 7:4551.

4. Steffens K. Competences, learning theories and MOOCs: recent developments in lifelong learning. Eur J Educ 2015; 50:41-59.

5. Abbasi AN, Kiziltan HS. Implementation of quality measures in site-specific multidisciplinary tumour boards. J Coll Physicians Surg Pak 2017; 27:388.
Muneeb Uddin Karim ${ }^{1}$, Andrea D'Aviero', Agha Muhammad Hammad Khan ${ }^{1}$ and Ahmed Nadeem Abbasi ${ }^{1}$

1 Department of Oncology, The Aga Khan University, Karachi, Pakistan

2 Department of Oncology, Fondazione Policlinico Universitario A. Gemelli IRCCS, Università Cattolica del Sacro Cuore, Rome, Italy

Correspondence: Dr. Muneeb Uddin Karim, Section of Radiation Oncology, Department of Oncology, The Aga Khan University, Stadium Road, Karachi, Pakistan E-mail: muneeb.uddin@aku.edu

Received: July 12, 2018; Accepted: September 07, 2018 ..........

\section{CORRIGENDUM}

This is with reference to the article titled "A Comparative Histological Study of Submucosal Gland Hypertrophy in Trachea of Mice", published in JCPSP 2018, Vol. 28 (3): 192-5.

It is to be informed to all that there has been an inadvertent error in mentioning the workplace of the third author, Dr Shazia Muazam, wherein, Rawal Institute of Medical Sciences (RIMS) has been published in place of Rawal Institute of Health Sciences (RIHS)

Hence, the following correction may please be noted:

\section{CORRECTION}

"Rawal Institute of Medical Sciences (RIMS)" mentioned at page number 192 as workplace of the third author may please be replaced as "Rawal Institute of Health Sciences (RIHS)". 